\title{
Eventcoin: Criptomoeda para Gamificação de Eventos
}

\author{
Alexandre Pereira Werner ${ }^{1}$, Cristiano Bertolini², \\ Sidnei Renato Silveira ${ }^{2}$, Joel da Silva ${ }^{2}$ \\ ${ }^{1}$ Curso de Bacharelado em Sistemas de Informação - UFSM - Universidade Federal de \\ Santa Maria - Campus Frederico Westphalen/RS \\ ${ }^{2}$ Departamento de Tecnologia da Informação - UFSM - Universidade Federal de Santa \\ Maria - Campus Frederico Westphalen/RS \\ xandewerner.2@gmail.com, cristiano.bertolini@ufsm.br, \\ sidneirenato.silveira@gmail.com, joel.silva@ufsm.br
}

\begin{abstract}
Resumo. Blockchains funcionam como um banco de dados distribuído em uma rede peer-topeer, onde cada usuário tem acesso a uma cópia atualizada de todo o histórico de transações realizadas. A utilização de um sistema baseado em blockchain garante confiabilidade, sem precisar de um mecanismo centralizador ou regulador. Nesse contexto, este artigo apresenta uma aplicação baseada em blockchains, combinando com o conceito de gamificação, para proporcionar uma nova maneira de validar, armazenar e distribuir certificados de eventos, tais como workshops, conferências e minicursos.
\end{abstract}

Palavras-Chave: Blockchain. Gamificação. Descentralização.

\begin{abstract}
Blockchains work as a distributed database in a peer-to-peer network, where each user has an authenticated copy of the transactions history access. The usage of a blockchain based system guarantee reliability without a centralized or regulator mechanism. This paper presents an application based on a blockchain, combining with the concept of gamification, to provide a new way to validate, store and distribute certificates in events such like workshops, conferences e curses.
\end{abstract}

Keywords: Blockchain, Gamification, Descentralization.

\section{Introdução}

Um dos problemas enfrentados atualmente por usuários, empresas e organizações é a necessidade de validação e acesso às informações de documentos públicos na Internet, e de meios que comprovem a existência e a originalidade dos arquivos digitais. Também, no caso de eventos, palestras e minicursos, é preciso buscar formas de atestar a presença dos inscritos nesses eventos. Uma das formas de disponibilizar e validar esses documentos seria por meio de mecanismos semelhantes aos utilizados pelas criptomoedas, e o incentivo ao uso poderia ser por meio de recompensas ou bônus. A tecnologia usada para a implementação de criptomoedas chama-se blockchain (Costa et al., 2018).

A Blockchain é a estrutura por trás do Bitcoin, a primeira criptomoeda funcional construída em uma rede distribuída, descentralizada e confiável, utilizando criptografia. A rede do Bitcoin é um ambiente onde os usuários podem transferir a posse de bens digitais (que funciona como uma espécie de moeda) entre eles, sem a necessidade de passar por nenhuma entidade verificadora, como bancos ou sistemas de pagamentos, 
pois todos os registros estão criptografados e disponíveis para auditoria, funcionando ao mesmo tempo como uma moeda e um sistema de pagamentos. A tecnologia que permite isso é o blockchain (Ulrich, 2017).

A gamificação consiste em adicionar atributos e aspectos de jogos para outras atividades que não sejam jogos, ou seja, uma maneira de criar motivação e incentivos para utilização de aplicativos ou serviços. Mesclar a capacidade do blockchain em armazenar e validar informações com o conceito de gamificação é uma maneira de criar uma aplicação que, ao mesmo tempo que incentiva os usuários a participarem e se engajarem nos eventos, também cria mecanismos para garantir a autenticidade e a propriedade dos certificados de participação nestes eventos.

Desta forma, o objetivo deste trabalho foi o de desenvolver uma plataforma de validação e armazenamento de certificados, utilizando os conceitos que permitiram o surgimento das criptomoedas, e que, aliando blockchains e o conceito de gamificação, permitiu criar uma forma de armazenar, distribuir e validar certificados de eventos como palestras, minicursos, seminários e eventos similares.

Para dar conta desta proposta, o artigo está organizado como segue. A seção 2 apresenta uma breve fundamentação teórica, destacando conceitos relacionados ao blockchain e gamificação. A seção 3 aborda alguns trabalhos relacionados. Na seção 4 são apresentados os detalhes da modelagem, implementação e validação da plataforma desenvolvida. Encerrando o artigo são apresentadas as considerações finais e as referências empregadas.

\section{Fundamentação Teórica}

Nesta seção são apresentados os principais conceitos para o desenvolvimento e implementação de um blockchain, envolvendo criptomoedas, blockchain e gamificação.

\subsection{Blockchain}

O Blockchain pode ser descrito como uma espécie de banco de dados distribuído em uma rede, onde todos os usuários têm acesso a uma cópia atual e fidedigna do histórico de transações da rede a todo instante. Os usuários dessa rede podem ser anônimos e não existir uma entidade controladora, como no caso do Bitcoin, formalizando, assim, uma rede blockchain pública, ou também pode-se construir um blockchain privado, onde os usuários são identificados e existirá uma entidade certificadora e controladora da rede. Os dados ficam organizados em blocos encadeados entre si, em uma estrutura de dados chamada Merkle Tree, e são criptografados com o uso do algo ritmo SHA-256, criando hashes que assinam digitalmente o conteúdo desses blocos, garantindo a imutabilidade da informação. A única forma de evitar a ausência de uma transação é estar ciente de todas as transações (Nakamoto, 2008), então a blockchain funciona como um livro que registra toda a informação nele contida, criptografando cada informação em cada bloco, e posteriormente, toda a estrutura, tornando-a assim, imutável. Aliando criptografia e estrutura de dados com uma rede distribuída e aberta, onde cada participante da rede possui uma cópia com todos os registros feitos na história da moeda, sendo facilmente editável e rastreável até o primeiro bloco da cadeia, o blockchain garante a integridade e segurança dos dados (Nakamoto, 2008). 
Por essas propriedades, essa tecnologia possui a capacidade única de garantir descentralização com segurança, sem a necessidade de confiança mas, sim, criando um ambiente onde tudo pode ser verificado e auditado. Sua aplicabilidade inicial era ser o livro-razão (ledger) do Bitcoin, para resolver o problema do gasto duplo de moedas virtuais, isto é, evitar que uma quantidade de moeda fosse gasta duas vezes pelo mesmo usuário (Nakamoto, 2008). Com isso, podemos dizer que a informação contida em um blockchain pode ser descrita como um bem não fungível, ou seja, temos um bem digital não duplicável, cuja propriedade pode ser transmitida de uma pessoa para outra e há um consenso público sobre essa propriedade.

O blockchain pode ser visto como um framework, composto por vários pequenos componentes, que somados, se tornam um todo compreensível. Nas próximas seções são abordados os seguintes componentes: hash, estrutura de dados, provas de trabalho, consenso, transações, mempool, assinaturas, chaves pública e privada, carteiras (wallet) e redes.

\subsection{Função Hash}

Uma função Hash é um algoritmo que mapeia dados de comprimento variável para dados de comprimento fixo, ou seja, consiste em usar, como entrada, diferentes strings, com qualquer tamanho, e ter como saída uma string de tamanho fixo. No contexto das criptomoedas, as transações são usadas como entrada e processadas por um algoritmo de hashing, o SHA-256, que produz uma saída, que é um hash de tamanho fixo previamente estabelecido.

A Figura 1 apresenta a estrutura de um blockchain. Em uma lista encadeada temos apenas uma série de blocos, onde cada um contém um ponteiro para o bloco anterior, mas em um blockchain esse ponteiro é substituído por um hash pointer. Então, cada bloco não contém apenas o endereço do anterior mas, também, um resumo criptográfico do conteúdo, o que nos permite verificar que os dados não foram alterados. Assim, no topo de cada bloco fica armazenado esse hash pointer, que aponta para o bloco mais recente (Narayanan et al., 2016).

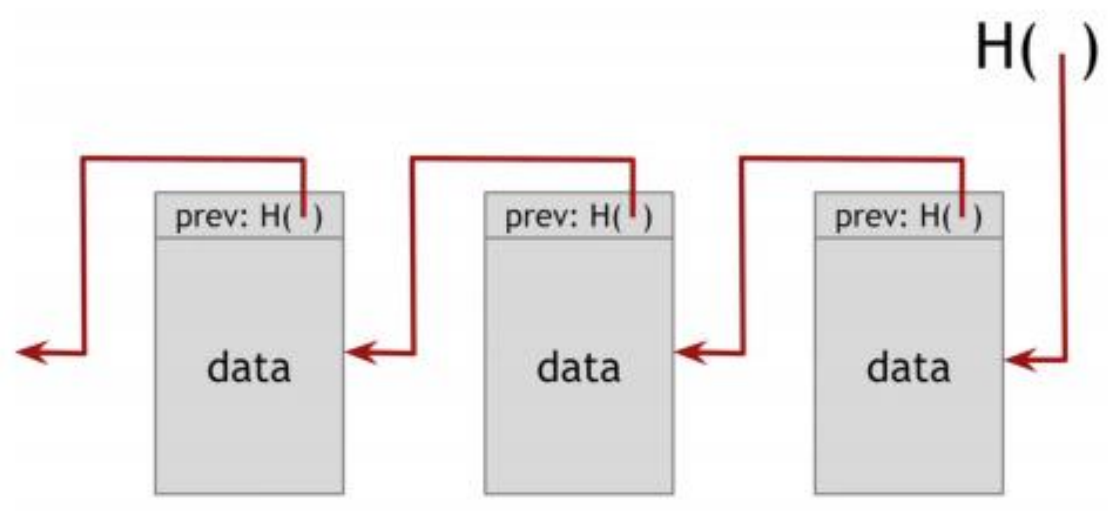

Figura 1. Blockchain: lista encadeada construída com hash pointers ao invés de somente ponteiros

Fonte: (Narayanan et al., 2016) 


\subsection{Estrutura de dados - Merkle Tree}

Merkle Tree (Agner, 2016) é uma estrutura de dados que contém uma árvore de informações resumidas, onde cada bloco guarda um resumo sobre um pedaço de dado maior. Esse tipo de estrutura é usado em sistemas de verificação, tanto em estruturas simples como a blockchain, como em estrutura mais complexas, como bancos de Dados NoSQL e programas usados para controle de versão, como Git 4 e Mercurial 5. Resumidamente, Merkle Trees são estruturas de dados utilizadas para criar um resumo de dados, com integridade criptograficamente verificável de forma eficiente quando em poder da raiz de Merkle - que vai do cabeçalho de cada bloco - e de um caminho de Merkle (Agner, 2016).

Seu funcionamento pode ser descrito da seguinte forma: supondo que se queira checar se um arquivo está íntegro e não foi modificado em uma rede, em vez de se enviar uma cópia completa dos arquivos de A para B, para efetuar testes de sua validade, envia-se um hash do arquivo de $\mathrm{A}$ até $\mathrm{B}$, e esse hash é comparado com a informação armazenada no nó root da estrutura. Com essas características é possível verificar toda uma cadeia de blocos somente conferindo os cabeçalhos (headers) de cada estrutura (Nakamoto, 2008). Na Figura 2 vemos uma representação dessa estrutura. Os blocos são agrupados em pares de dois blocos cada, e para cada dupla, é construída uma estrutura de dados que contém dois hash pointers, um para cada bloco. Com essa estrutura, construímos o próximo nível da estrutura, formando uma árvore. E, para cada dupla, se cria uma nova assinatura e uma nova estrutura, assim sucessivamente, até chegarmos ao bloco principal da árvore. Com isso temos a possibilidade de verificar cada hash pointer da estrutura, permitindo, assim, a verificação de que nenhum bloco foi alterado, pois se algum bloco for alterado, seu hash será modificado, e não será mais o mesmo que o do bloco superior (Narayanan et al., 2016).

Dentro do contexto da blockchain, é essa estrutura de dados que permite a transparência das operações, garantindo que é possível consultar toda a informação referente a cada bloco e, consequentemente, todas as transações da rede, desde o seu início.

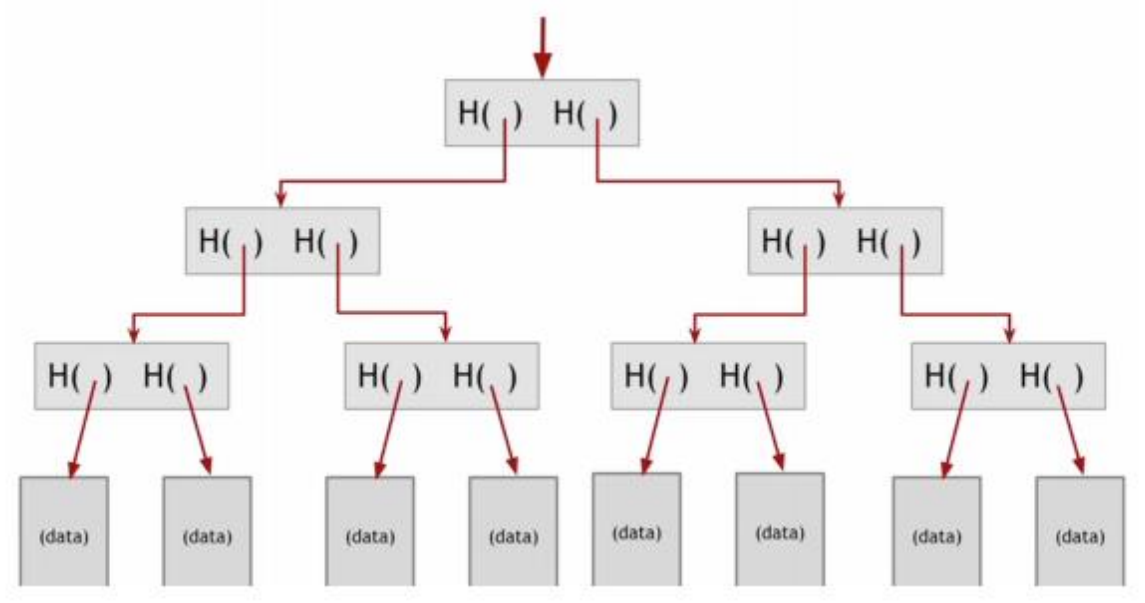

Figura 2. Merkle Tree

Fonte: (Narayanan, et al., 2016) 


\subsection{Prova de Trabalho - Proof of Work}

Para o funcionamento de um blockchain e sua rede descentralizada, é necessário existir consenso sobre o estado dos registros contidos no blockchain. Para chegar nesse consenso, existem algumas técnicas possíveis para se garantir que aquele registro é válido. A solução para isso no blockchain do Bitcoin foi encontrada por meio da prova de trabalho (proof-of-work) (Nakamoto, 2008). Uma prova de trabalho é um desafio criptográfico utilizado para garantir que um dos participantes da rede, um nodo (node), realizou uma certa quantidade de trabalho. O Bitcoin utiliza uma prova de trabalho baseada no modelo de Adam Back (Nakamoto, 2008).

Outras aplicações que utilizam blockchain implementam variações deste modelo, mas que atendem dois princípios: 1) garantir que o nó realizou uma quantidade predefinida de processamento e 2) garantir que a prova entregue é verificável. Tipicamente, uma prova de trabalho é um processo probabilístico, e a probabilidade de sucesso depende da dificuldade estabelecida. No Bitcoin, a prova de trabalho é encontrar um valor chamado nonce, cujo duplo hash SHA256 desse valor com a Merkle Tree seja igual ou menor que um parâmetro inicial, no caso do Bitcoin, a transação.

\subsection{Prova de Existência - Proof of Existence}

Em contrapartida à prova de trabalho, que gera um gasto de energia muito grande para solucionar os problemas matemáticos, foram desenvolvidas outras formas de gerar consenso na rede. Uma das mais utilizadas é a prova de participação (proof of stake) na rede Ethereum (Buterin, 2013). Entretanto, a que mais se encaixa mais nesta proposta é a prova de existência (proof of existence).

A prova de existência consiste em usar hashes criptográficos para comparar e atestar que a informação que consta no blockchain é a mesma que a de um determinado arquivo. Com isso conseguimos demonstrar a propriedade de determinado bem digital sem revelar seu conteúdo, e determinamos a existência do documento sem uma entidade centralizadora e ainda garantimos a integridade do documento, pois a menor alteração resulta em um hash diferente, gerando uma inconsistência.

\subsection{Consenso}

Existem vários desafios para a elaboração de uma moeda digital, e o mais complexo de se resolver era evitar o gasto duplo, que foi evitado com a estrutura de dados do blockchain. Outro desafio significativo era o de criar incentivos para a utilização da moeda, o que foi resolvido pela prova de trabalho. Entretanto, quando dois blocos realizarem a prova de trabalho com uma diferença de tempo pequena, e conseguirem transmitir essa informação na rede, ocorrerá uma disputa. Esse evento é chamado de Corrida de Blocos. O protocolo da rede resolve esse problema aceitando o bloco com maior tamanho, o que chamamos de Regra da Maior Cadeia (Longest Chain Rule) (Nakamoto, 2008). Sendo assim, todo o bloco aceito na rede é ligado criptograficamente ao anterior, sendo possível percorrer todos os blocos até o primeiro bloco da cadeia, conhecido como bloco genesis. 


\subsection{Transações}

As transações são estruturas de dados que codificam a transferência de valor entre os participantes do sistema. Em suma, uma transação envolve uma assinatura digital do emissor (que detém o ativo) e o endereço do receptor, além de entradas e saídas com o valor, ou outra informação transacionada, ou seja, cada transação é determinada com seu valor de hash representando um identificador de transação e um conjunto de entradas e saídas. Cada saída da transação só pode ser usada uma vez como uma entrada em todo o blockchain (Tschorsch; Scheuermann, 2016). Para se manter a consistência dos dados, a única maneira de se confirmar a presença de uma transação na rede é estar ciente de todas as transações (Nakamoto, 2008).

\subsection{Mempool}

O Mempool (Antonopoulos, 2014) é um componente essencial de uma rede descentralizada, pois é nessa estrutura que as transações ficam esperando sua validação, ou seja, o mempool é uma lista temporária de transações não-confirmadas. Os nós usam esse pool para manter um acompanhamento das transações que são conhecidas pela rede, mas que ainda não foram incluídas no blockchain. No mempool, as transações são recebidas e verificadas, sendo adicionadas ao pool de transações e transmitidas aos nodos vizinhos, para serem propagadas para a rede (Antonopoulos, 2014).

\subsection{Assinatura, Chaves e Carteiras}

A posse de um ativo ou informação no contexto do blockchain se dá por meio de chaves assimétricas e assinaturas digitais. Essa identidade funciona como uma assinatura manual, ou seja, só você pode produzí-la, mas qualquer um pode verificá-la. Então é criada uma chave privada, que é nada mais do que um número escolhido ao acaso. A posse e o controle da chave privada são tudo o que o usuário precisa para controlar todos os registros associados ao endereço correspondente.

A chave privada é usada para criar assinaturas que são necessárias para se comprovar a posse dos registros usados em uma transação. A chave privada deve sempre ser mantida em segredo, pois revelá-la a terceiros é equivalente a fornecê-los o controle sobre todos os registros protegidos por aquela chave. Entretanto, a chave pública deve ser revelada para qualquer um dos usuários da rede que queiram atestar a autenticidade da assinatura (Antonopoulos, 2014).

\subsection{Rede}

A estrutura do blockchain do Bitcoin foi pensada como uma arquitetura de rede peer-topeer (Nakamoto, 2008), e dessa forma, todos os participantes se comunicam diretamente entre si, atuando como cliente e servidor. Por meio dessa arquitetura é que se alcança a descentralização, onde toda a rede funciona de forma independente, sem uma unidade central, mesmo que alguns participantes saiam dela, a operação continua, e todos os usuários têm acesso a todas as transações que existem na rede a todo o momento (Nakamoto, 2008). A Figura 3 representa uma comparação entre três tipos de redes: centralizada, decentralizada e distribuída. 


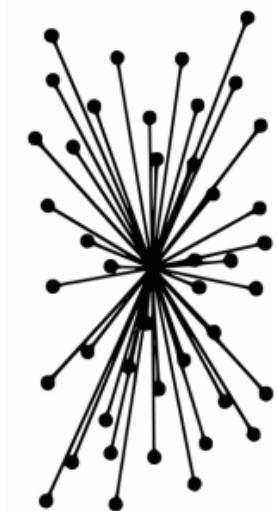

Centralized

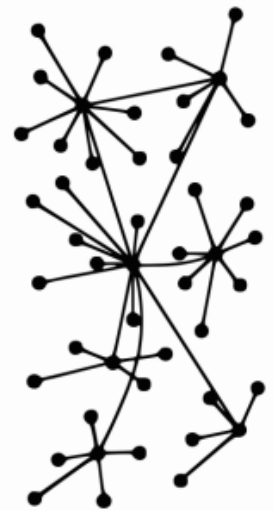

Decentralized

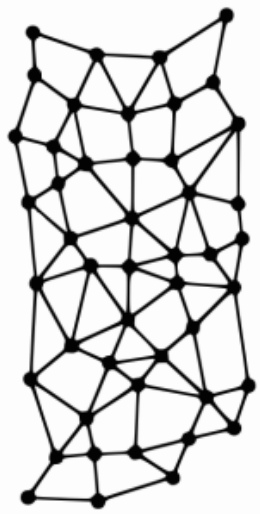

Distributed

Figura 3. Rede Blockchain

Fonte: Dos autores, 2020, adaptado de (Baran, 1964)

\subsection{Tipos de Blockchain}

Podemos classificar algumas implementações de blockchain conforme a permissão dada aos usuários em utilizá-la (Walport, 2016):

- Blockchain Público - Em um blockchain público todos os usuários podem ler e enviar transações, ou seja, qualquer um pode participar dessa rede por meio dos mecanismos criadores de consenso na rede (proof-of-work), pois este tipo de blockchain não requer permissão de terceiros (Bitfury Group, 2015b). Todas as transações são públicas e os usuários podem permanecer anônimos. Por exemplo, as criptomoedas Bitcoin e Ethereum são implementadas desta maneira;

- Blockchain privado - Um blockchain privado funciona como um banco de dados convencional. O conteúdo é armazenado de forma que somente usuários identificados e com permissão podem acessá-lo, por meio de um controle de verificação e permissões. Mas, ao contrário de um banco de dados tradicional, os dados não podem ser alterados e todas as transações são verificadas por um mecanismo de consenso que é estabelecido pelos administradores da rede (Bitfury Group, 2015a);

- Blockchain semi-privado - Parte do blockchain é privado e parte é público. A parte privada é controlada por um grupo de usuários individuais, enquanto que a parte pública é aberta para a participação de qualquer um (Bashir, 2017);

- Sidechains - Nessa categoria, as informações contidas no blockchain podem ser movidas para outro blockchain. O uso de sidechains é mais comum na criação de novas criptomoedas ou em estruturas de apoio (Bashir, 2017).

\subsection{Serviços, Atores e Papéis}

Na Figura 4 temos uma visão dos componentes empregados pelos usuários para interagir com a tecnologia: o ator é representado por: 'Usuário': Ator que interage com o blockchain criando transações. Os papéis são: 
- Criação de blocos: Usuário ou sistema responsável pela validação das transações, construir novos blocos, assinar ou transmití-los para o blockchain. Também é responsável pelo consenso na rede, garantindo confiança entre as partes envolvidas.

Os serviços são:

- Criação de Transações: é o meio pelo qual um usuário pode adicionar informação para o blockchain. Podem ser pagamentos, recebimentos, contratos, permissões, etc;

- Submissão de Transação: consiste em enviar a transação e transmití-la para toda a rede;

- Validação de bloco: é um serviço usado pelos nodos para validar os novos blocos adicionados ao blockchain;

- Geração de Blocos: é dividida em pequenos serviços que são usados de formas diferentes, dependendo do tipo de blockchain. Um minerador em um blockchain público irá usar um bloco gerador, em um blockchain privado, um serviço pode criar o bloco e outro o assina;

- Permissões de acesso ao blockchain: é uma característica exclusiva dos blockchains privados, onde nodos administrativos garantem permissão aos usuários identificados da rede. Também pode ser usada para criar tokens.

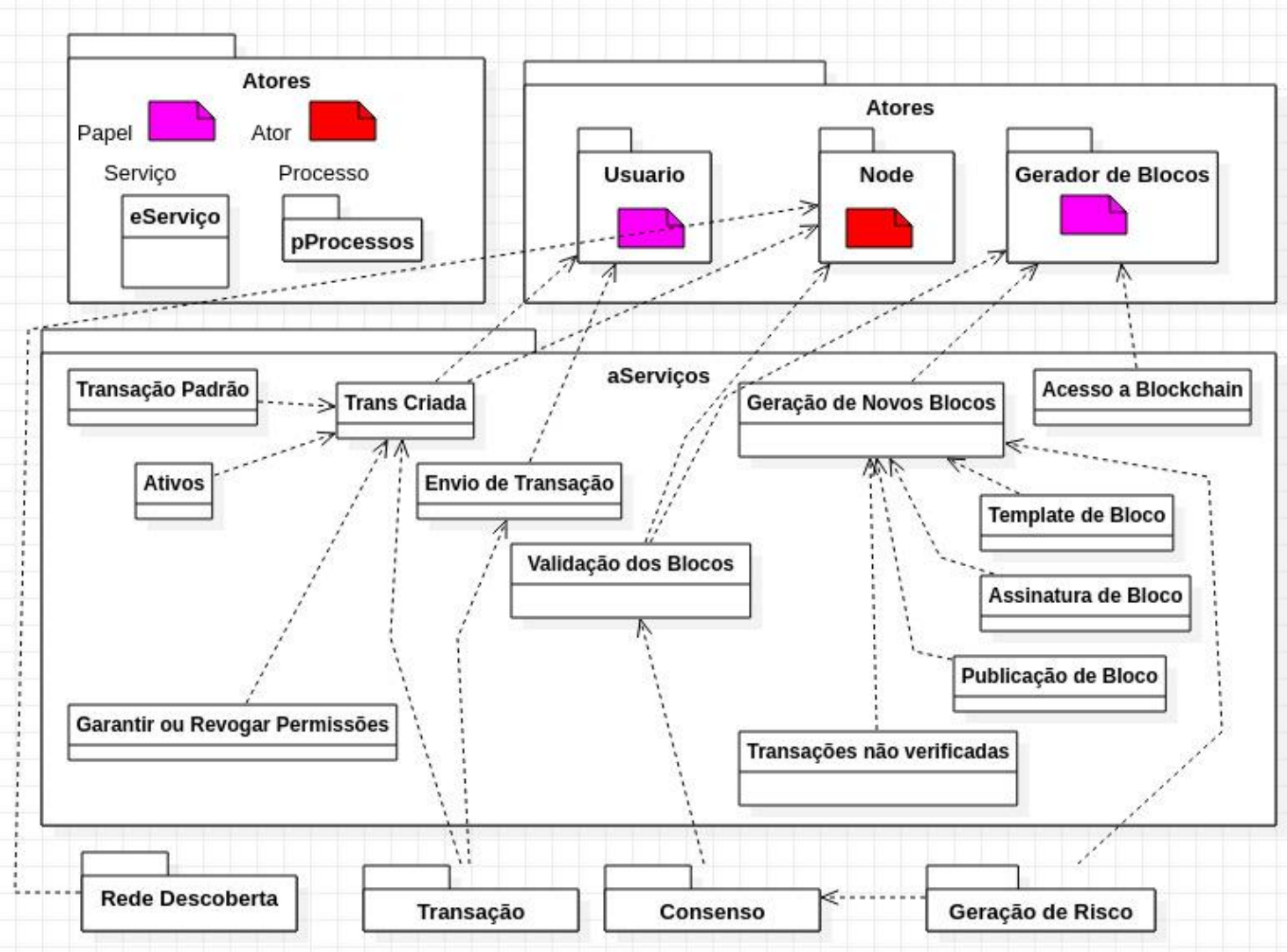

Figura 4. Processos - Rede Blockchain

Fonte: Adaptado de (Ellervee et al., 2017) 


\subsection{Gamificação}

A gamificação consiste em adicionar atributos e aspectos de jogos para outras atividades que não sejam jogos. Ao se utilizar esse conceito, procura-se atingir um público maior e familiarizá-los com os conceitos ou proposta apresentada. Também podemos considerar a gamificação como um processo para reforçar serviços, aliando uma experiência de jogos, para dar suporte às atividades dos usuários (Huotari; Hamari, 2017).

Em termos gerais, a gamificação é uma maneira de criar motivação e incentivos para utilização de aplicativos ou serviços. Mesclar o blockchain com a gamificação é uma maneira de ajudar aos usuários da Eventcoin a manterem, protegerem e validarem suas informações, enquanto criamos um incentivo para mantê-los usando a proposta. Quando eventos são gamificados, temos um grande resultado, uma sinergia, atraindo tanto a audiência quanto jogadores, o que beneficia os organizadores e o evento (Moise; Cruceru, 2014).

Com isso, queremos criar uma abordagem diferente. Ao invés de utilizar uma prova de trabalho matemática, como no caso do Bitcoin, para atestar que determinada moeda ou transação foi feita por determinado agente ou minerador, nesta proposta o aluno (ouvinte, participante) terá de ir até o evento, cumprir uma determinada tarefa, que é a de assistir o evento até o fim e, então, irá receber sua recompensa, o certificado.

\section{Trabalhos Relacionados}

Esta seção apresenta alguns trabalhos relacionados à proposta, envolvendo blockchain, certificados e gamificação de eventos. $\mathrm{O}$ trabalho mais próximo à proposta deste artigo é o Blockcerts, desenvolvido pelo MIT (Media Lab and Machine Learning) (Blockcerts, 2016).

Nessa plataforma, os alunos submetem seus certificados para serem digitalmente assinados, validados e disponibilizados de forma descentralizada. Essa proposta é composta de quatro pilares: (1) Emissor (Issuer) A universidade cria um certificado digital que contém informações sobre os usuários e os registra no blockchain do Bitcoin; (2) Certificado (Certificate): Certificados são recompensas; (3) Verificador (Verifier): Qualquer pessoa pode, sem depender do emissor, verificar se um certificado não foi adulterado, foi emitido por uma instituição específica e emitido para um usuário específico. (4) Carteira (Wallet): Os alunos podem armazenar de forma segura seus certificados e compartilhá-los com quem quiserem.

Após a validação por um verificador, os certificados são registrados no blockchain, assinados digitalmente, validados e por meio de uma chave pública e do endereço imutável no blockchain, e ficam disponíveis para serem compartilhados com qualquer instituição ou pessoa. A proposta do Blockcerts optou por usar o blockchain do Bitcoin por ser mais popular e, consequentemente, mais usada e testada (Blockcerts, 2016).

Enquanto a proposta do Blockcerts foca apenas em criar uma validação e registro de um certificado já existente, a proposta aqui apresentada (Eventcoin) é a de criar uma plataforma que envolva todos os elementos da emissão de um certificado, 
começando com o controle da presença dos espectadores do evento, passando pela emissão e posterior verificação da origem e validade do certificado.

Kano e Nakajima (2017) apresentam uma solução para o problema da concentração excessiva do poder computacional da rede do Bitcoin em poucos agentes, deixando o processo de mineração do Bitcoin muito centralizado. Este trabalho utiliza o conceito de gamificação. Os autores buscaram encontrar uma nova forma que permitisse uma maior descentralização do poder computacional da rede, atraindo os usuários com incentivos, para estes desempenharem o trabalho de aprovação de novos blocos na rede. Por meio do uso de gamificação, houve um aumento do engajamento de usuários no processo, mas ainda é necessário aumentar o poder computacional para manter a rede funcionando. Com esse trabalho, vemos que a gamificação é uma das alternativas para se construir uma rede descentralizada e com engajamento dos usuários. Entretanto, ao contrário desta proposta, na Eventcoin a gamificação será usada para os usuários se engajarem nos eventos, e não na mineração de novas unidades de criptomoeda.

Duas outras plataformas também estão relacionadas com a proposta: Original My (Original My.COM, 2019), empresa brasileira de registro de documentos digitais, contratos e identidade de pessoas no blockchain e a Proof of Existence (ProofofExistente.COM, 2019), primeiro serviço desenvolvido para fins notariais utilizando o blockchain do Bitcoin. Nas duas propostas utiliza-se a estrutura existente do Bitcoin para armazenar o hash de um documento e atestar a data de inclusão usando um carimbo timestamp. Ambas as propostas armazenam essas informações em um script chamado OP RETURN. Nesse script normalmente são incluídas informações não relacionadas com operações financeiras. Nessas propostas, apenas se faz o registro de um documento em uma rede blockchain já existente e, diferentemente da proposta do BlockCerts, pode-se fazer de qualquer tipo de documento, não apenas certificados, além de se reconhecer identidades digitais.

\section{EventCoin}

A EventCoin consiste em uma solução de armazenamento e validação de certificados, usando blockchain, aliada ao conceito de gamificação, trazendo uma nova forma de validar, armazenar e distribuir os certificados de eventos. A proposta compreende um blockchain permissionado, onde os usuários são identificados e a geração de certificados é supervisionada por um ente garantidor da emissão, no caso, o coordenador de cada evento. Em um blockchain permissionado, a criação de novos blocos não está ligada a uma prova de trabalho que exija mineração (BitFury Group, 2015a).

Quando um aluno se cadastra no evento, minicursos, semana acadêmica ou palestras, entre outros eventos, a sua inscrição será equivalente a criar uma transação em espera, que no dia do evento será adicionada a um bloco. A Figura 5 apresenta uma visão geral da EventCoin. Quando um aluno se cadastrar em algum evento, é gerada uma lista de transações, com uma transação única para cada usuário. Este passo é descrito no Item 2 da Figura 5. Quando o evento ocorrer, cada aluno deverá ter sua wallet, que garantirá sua identidade por meio do par de chaves públicas e privadas confirmando, assim, que aquela transação é sua. Após essa etapa, sua transação será registrada em um bloco de transações referentes àquele evento no blockchain. Após é 
enviado para o aluno o endereço público de sua credencial no blockchain, que será validado pela comissão organizadora do evento.

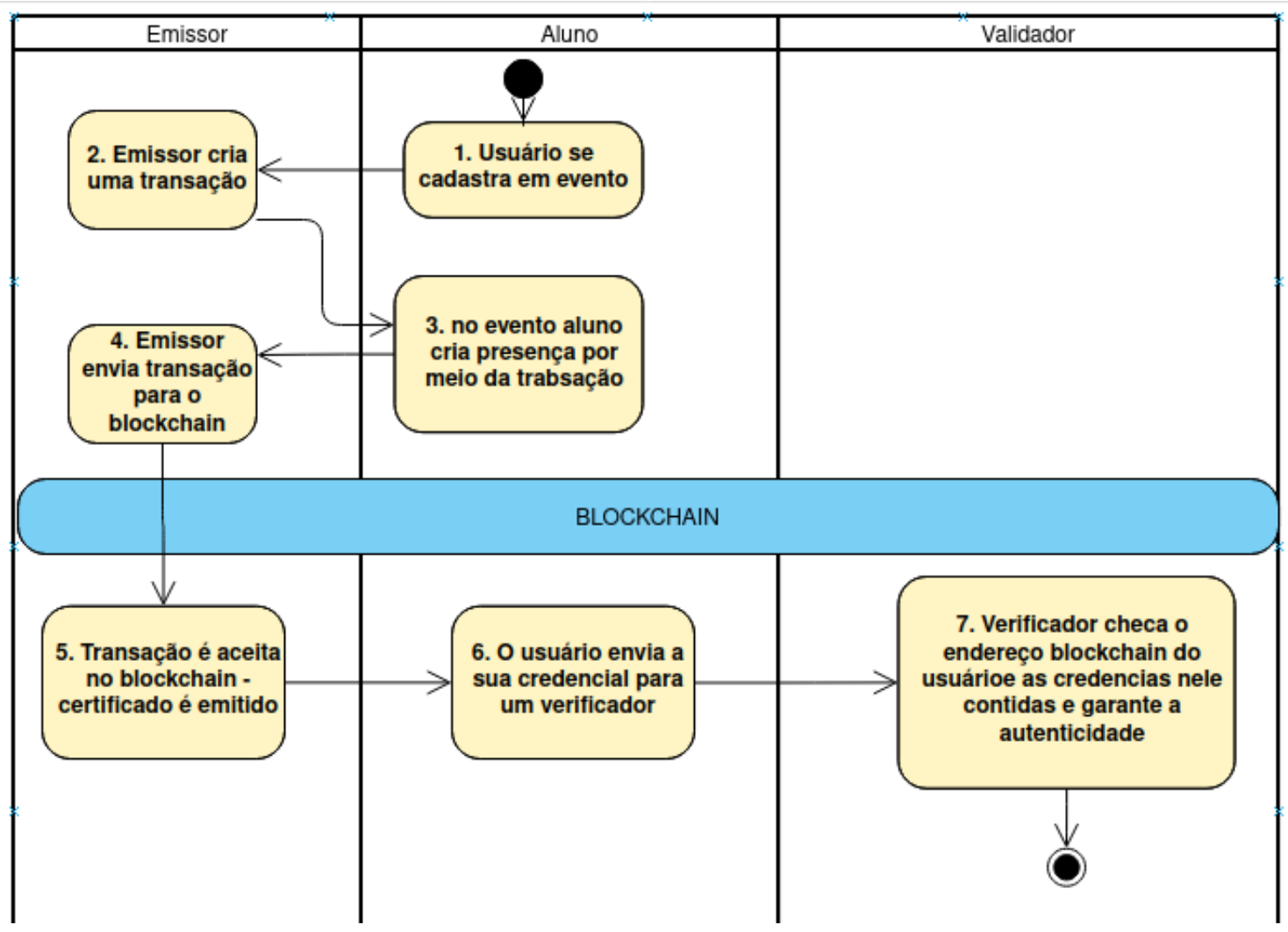

Figura 5 - Proposta Detalhada

Fonte: Dos autores, 2020

\subsection{EventCoin: Tecnologias e Arquitetura}

Esta seção apresenta as tecnologias utilizadas no desenvolvimento da aplicação. Também é apresentada a arquitetura escolhida para a comunicação entre a aplicação e os usuários. Para que o blockchain venha a ter utilidade, a informação nele contida deve ser acessível por outras aplicações e pela rede de usuários. Para esta aplicação ser acessível, escolhemos utilizar a tecnologia dos Web Services para a comunicação entre a aplicação e os usuários. Sendo o blockchain uma forma de armazenar dados, o web service é utilizado como uma ponte entre o usuário e a informação.

Para o desenvolvimento da aplicação foram escolhidas tecnologias já adaptadas ao contexto de desenvolvimento web. A linguagem de programação principal deste projeto foi Javascript. A escolha se deu por esta linguagem ter um ecossistema de frameworks muito maduro e robusto, já adaptado para trabalhar com web services e blockchain. A linguagem JavaScript foi escolhida para esse trabalho por ser largamente utilizada, com uma variedade grande de bibliotecas prontas para trabalhar tanto com Web Services e com as API's (Application Program Interface) dos principais blockchains existentes (Burnett, 2018). Javascript é uma linguagem de programação funcional interpretada, capaz de reproduzir os princípios da programação orientada a 
objetos. Também é uma linguagem simples, expressiva e de fácil manutenção (Crockford, 2008).

O NodeJS (Pereira, 2018) é uma plataforma construída sobre o motor JavaScript do Google Chrome, para facilmente construir aplicações de redes rápidas e escaláveis. Node.js usa um modelo de entrada/saída direcionada a evento não bloqueante, o que o torna leve e eficiente, ideal para aplicações em tempo real, com troca intensa de dados por meio de dispositivos distribuídos. Sua arquitetura totalmente non-blocking thread (não-bloqueante), apresentando um bom desempenho em relação ao consumo de memória e utilizando ao máximo e de forma eficiente o poder de processamento dos servidores, principalmente em sistemas que produzem uma alta carga de processamento. Usuários de sistemas Node estão livres de aguardarem por muito tempo o resultado de seus processos e, principalmente, não sofrem com deadlocks no sistema, porque nada bloqueia em sua plataforma. Além disso, desenvolver sistemas nesse paradigma é simples e prático (Pereira, 2018). Por ser um framework muito utilizado, com uma comunidade ativa de usuários, e com essas propriedades, foi escolhido para ser a plataforma de desenvolvimento da aplicação.

O Express.js (Pereira, 2018) é um framework para o desenvolvimento de aplicações web de grande escala, facilitando o desenvolvimento. Suas principais características são o suporte ao modelo MVC (Model-View-Controller), integração com SQL (Structured Query Language) e NoSQL, roteamento de URL's (Uniform Resource Locator) e call-backs, além de ser compatível com o NodeJS (Pereira, 2018). O LevelDB é um datastore embarcado baseado em pares chaves-valor, cujos elementos são arrays de bytes arbitrários, ordenados por chave (o mecanismo de ordenação pode ser redefinido). O datastore é usado como uma biblioteca em aplicações, e o seu funcionamento é simples. Cada base de dados é um conjunto de arquivos em um diretório e as operações básicas são Put (chave,valor), Get (chave) e Delete (chave). No LevelDB, os dados são comprimidos automaticamente, usando uma biblioteca de compressão de alto desempenho. O LevelDB é otimizado para operações de escrita em lote. Ele acumula várias mudanças de forma ordenada em memória e as escreve para o disco quando um tamanho máximo configurável é atingido. O resultado é um excelente desempenho de escrita de dados (Avram, 2011).

\subsection{Funcionalidades do EventCoin}

Esta seção apresenta as funcionalidades da aplicação implementada em detalhes, e a maneira como as funcionalidades foram desenvolvidas, mostrando trechos do códigofonte e uma visão geral das requisições realizadas, temos como compreender melhor o funcionamento da aplicação. A Figura 6 apresenta uma visão geral da implementação da aplicação (API implementada), utilizando o diagrama de sequência, que mostra a ordem na qual as solicitações entre objetos são executadas (Gammat et al., 1995), demonstrando como o usuário envia uma requisição, e como a transação é tratada dentro da API, passando pelo mempool até ser aceita no blockchain. 


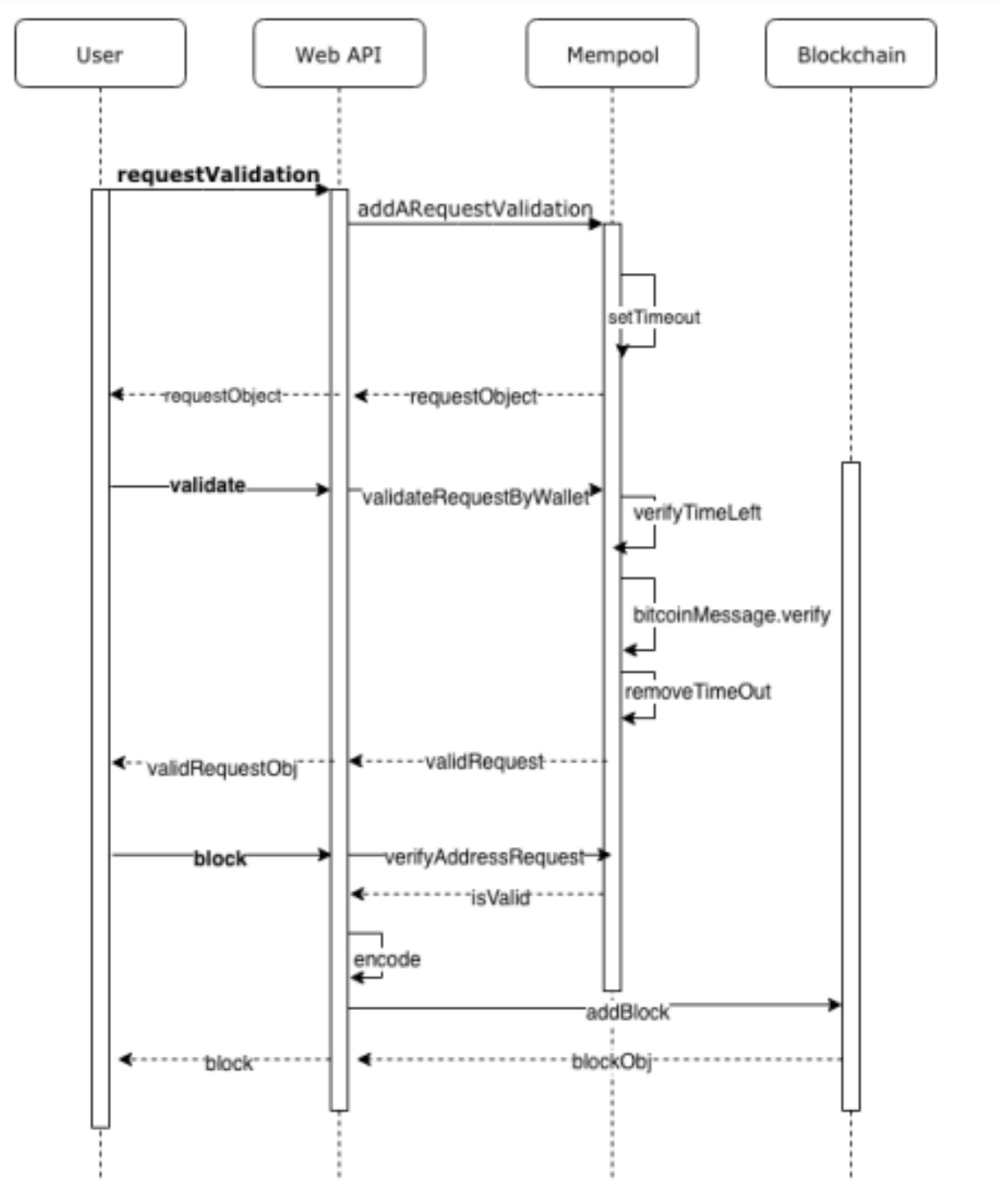

Figura 6. Implementação detalhada da API

Fonte: Dos autores, 2020.

\subsubsection{Aplicação}

A aplicação é formada por duas partes principais (i) o código que é executado no servidor, e que faz a inserção, validação e seleção de blocos; (ii) e a web API, que expõe os métodos para quem os consome, no caso, as carteiras e outros membros da rede. A Figura 7 apresenta a estrutura de um bloco com seu construtor.

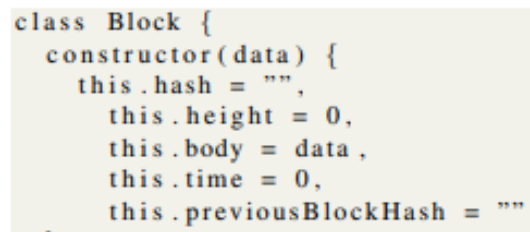

Figura 7. Estrutura de um Bloco

Fonte: Dos autores, 2020. 
Um bloco é formado pelas seguintes propriedades:

- Hash: é o resultado do hashing da informação do bloco, ou seja, é feito um hash de todo o conteúdo do bloco, sendo assim impossível de se alterar o conteúdo do bloco sem alterar seu hash, o que causará inconsistência quando comparado com o próximo hash da cadeia de blocos, como fica mais claro na Figura 8, linha 11;

- A propriedade height se refere ao tamanho do bloco, indicando a localização de um bloco no blockchain, ou seja, o número de blocos que precedem um bloco específico em uma cadeia de blocos. Por exemplo, o bloco da genesis tem uma altura de zero porque o bloco zero o precedeu;

- Na propriedade body podemos adicionar qualquer informação. No caso do blockchain da Eventcoin, aqui guardamos as informações referentes ao evento;

- A propriedade time guarda o carimbo timestamp do momento em que o bloco foi criado e o previousBlockHash guarda o hash do bloco anterior. A Figura 8 apresenta como um bloco é inserido no blockchain. Na linha 11 vemos como é realizado o hash do bloco, chamando a função SHA256 e passando o próprio bloco como parâmetro, ou seja, todo o conteúdo está sendo criptografado. Com isso, temos a imutabilidade do bloco, pois uma vez salvo na cadeia de blocos, o próximo aponta para o hash do momento da inclusão e, se houver uma tentativa futura de alterar os dados deste bloco, irá resultar em um hash diferente do salvo no próximo bloco, acusando assim, a inconsistência. Na linha 8 se procura o bloco anterior, para que possamos adicionar o hash do seu bloco na propriedade previousBlockHash.

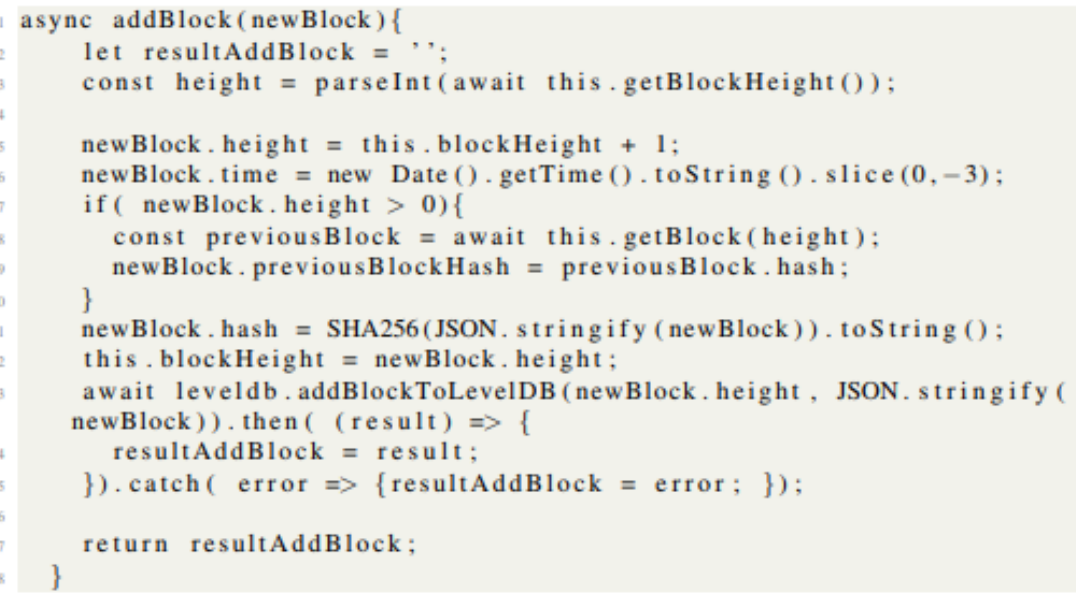

Figura 8 - Adicionando um Bloco

Fonte: Dos autores, 2020.

A Figura 9 apresenta o código necessário para validar um bloco que já está inserido na cadeia de blocos. Em um primeiro momento é selecionado do banco de dados (getBlock - linha 2) o bloco que se quer validar; na linha 3 salvamos o seu hash em uma variável (blockHash) e, na linha 5, utilizando a função SHA256, criamos um novo hash do bloco em teste. A partir daí fazemos a comparação com o valor que está armazenado na variável blockHash. Se os dois valores forem iguais, o bloco é válido, ou seja, não houve alteração de nenhuma informação. Do contrário, temos uma inconsistência: houve uma tentativa de se alterar os valores do bloco que já foi confirmado na cadeia de blocos. 


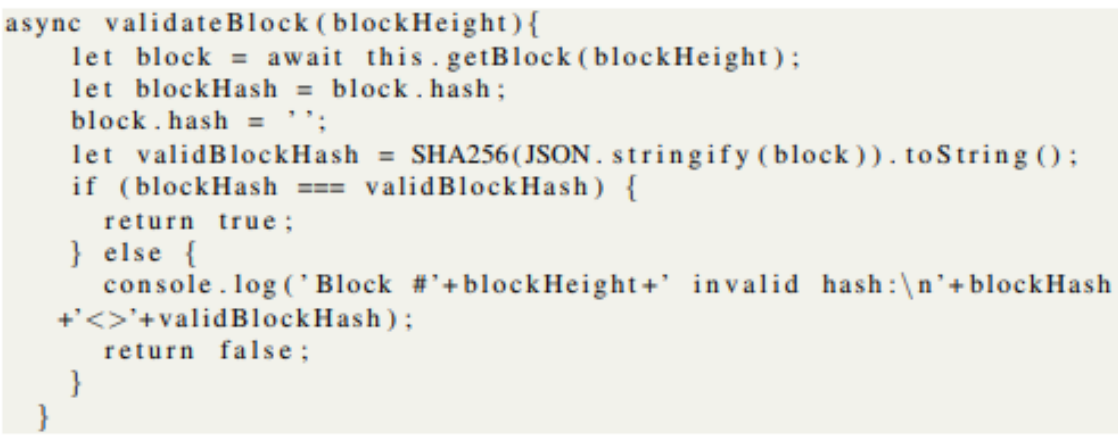

Figura 9 - Validação de um Bloco

Fonte: Dos autores, 2020.

Uma Web API é uma espécie de interface que contém uma quantidade de funções que permite que programadores acessem determinadas funcionalidades de uma aplicação, sistema operacional ou outros tipos de serviços. Neste trabalho foi construída uma API para que pudéssemos acessar as funcionalidades do blockchain em diferentes contextos. A visão geral desta funcionalidade está exposta na Figura 6. Em nossa API temos os seguintes métodos:

- validateAddressParameter: Validação dos parâmetros de endereço;

- validateSignatureParameter: Validação da assinatura;

- validateNewCertRequest: Validação para inclusão de um novo certificado;

- app.get('lock/:height): Descobrir o tamanho de um determinado bloco;

- app.get('cert/address:address): Acessar o endereço de um determinado certificado;

- app.get('/cert/hash:hash): Acessar o hash de um determinado certificado;

- app.post('/block): Adicionar um novo bloco, contendo transações, onde cada transação contém o hash de um certificado.

O usuário inicia uma transação, e a mesma é validada pelo método requestValidation, que faz a comunicação entre o usuário e a web API que, por sua vez, irá aguardar a resposta do mempool. Na Figura 10 vemos uma requisição sendo feita à web API.

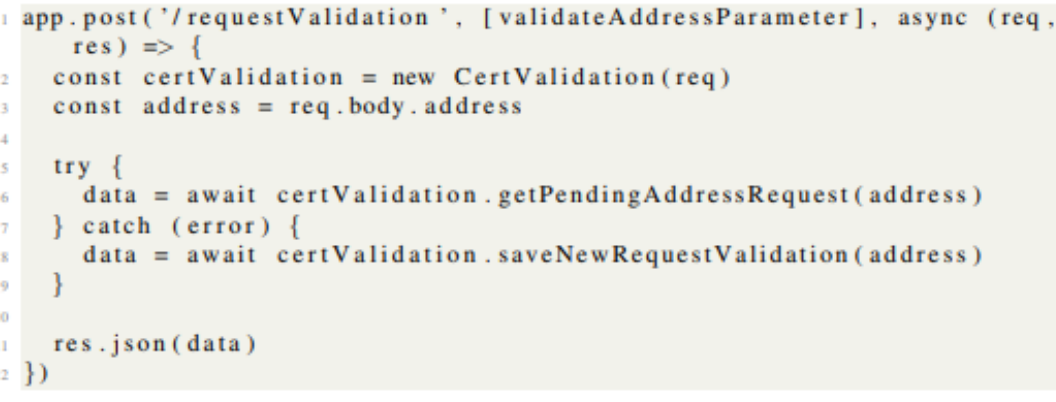

Figura 10 - Realização de uma Requisição para a API Implementada

Fonte: Dos autores, 2020. 
A Figura 11 apresenta um arquivo JSON (JavaScript Object Notation) que contém as informações de um certificado salvo, o conteúdo da propriedade body de um bloco.

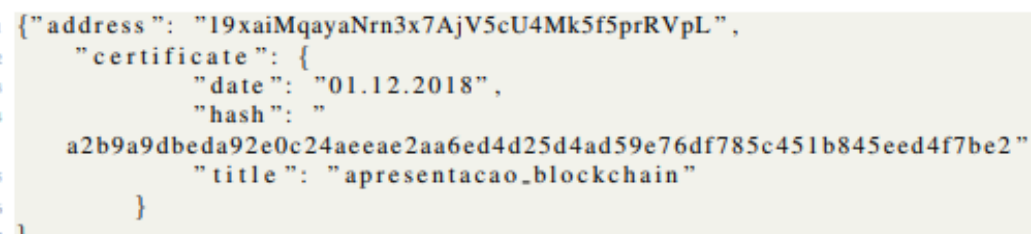

Figura 11 - Informações de um Certificado Salvo

Fonte: Dos autores, 2020.

\section{Considerações Finais}

Este trabalho apresentou um estudo sobre a origem, conceitos e utilização do blockchain, e uma proposta de desenvolvimento de uma criptomoeda para armazenar, compartilhar e verificar certificados dos alunos. Com essa abordagem, quer se demonstrar a versatilidade do blockchain e sua capacidade de ser ajustada aos mais variados contextos, seja na criação de uma moeda descentralizada, seja na criação de redes abertas, ainda descentralizadas, mas com usuários identificados e entidades certificadoras.

Para uma maior compreensão das técnicas utilizadas para se construir um blockchain e garantir suas características, foram descritos os componentes desta tecnologia e, também, foi realizada uma comparação com o funcionamento do protocolo Bitcoin. Tendo como foco a estrutura de dados, uma introdução ao funcionamento dos hashes e das transações e, por fim, da cadeia de blocos. Também abordamos como utilizar os métodos existentes para inserir dados no blockchain. Na seção 4, foram apresentados os detalhes acerca da aplicação desenvolvida, exibindo exemplos partes do código-fonte, tanto do blockchain desenvolvido, bem como da web API para acesso externo. Com o protótipo desenvolvido conseguimos registrar um conjunto de transações em um bloco e atestar a validade e existência dos certificados de um determinado evento. Do ponto de vista tecnológico, um dos principais resultados deste trabalho foi a criação de um ambiente para que pudéssemos demonstrar a utilização do blockchain para finalidades não relacionadas a finanças e, também, disponibilizar um ambiente para validação de certificados, fornecendo mais segurança e confiabilidade para o processo.

A implementação de uma solução baseada em um blockchain é complexa. É preciso levar em conta várias áreas da computação, tais como redes de computadores, algoritmos, criptografia, estruturas de dados, bancos de dados e interfaces web.

Um dos pontos importantes na implementação é o consenso, o qual garante que os certificados sejam íntegros e não possam ser apagados. O consenso é garantido utilizando-se a prova de existência (Chopra et al., 2019), consistindo em provar a existência de um documento, no caso de um certificado, a partir de um dado instante. A ideia é incentivar instituições (especialmente as universidades) a terem essa responsabilidade de executarem esses nós, além de algumas entidades privadas que 
possam se beneficiar desses documentos, para que possamos ter uma forma de validar e certificar que esses títulos (certificados, atestados, comprovantes, entre outros) são verdadeiros, utilizando a menor quantidade possível de poder computacional. Como trabalho futuro, para o funcionamento do consenso de forma íntegra, esperamos ter verificadores que executam os nodes, mas com todos os usuários podendo baixar e conferir os dados.

Além disso, também destacamos, como trabalhos futuros: desenvolvimento de wallets próprias; desenvolvimento de um ambiente de cadastro dos eventos e participantes, para que não seja necessária realizar manualmente uma carga inicial das transações para os participantes; descrição mais elaborada dos processos envolvidos em uma rede descentralizada utilizando blockchain; descrição mais aprofundada do processo de inserção de dados em um blockchain já existente.

\section{Referências}

Agner, M. (2016). "Bitcoin para Programadores". Instituto de Tecnologia e Sociedade do Rio. Disponível em: https://itsrio.org/wp-content/uploads/2018/06/bitcoin-paraprogramadores.pdf. Acesso em março, 2019.

Antonopoulos, A. M. (2014). "Mastering Bitcoin: Unlocking Digital CryptoCurrencies”. O’Reilly Media, Inc., 1st edition.

Avram, A. (2011). "LevelDB do Google: open source e alto desempenho com estruturas chave-valor". Disponível em: https:/www.infoq.com/br/news/2011/08/LevelDB. Acesso em maio, 2019.

Baran, P. (1964). "On Distributed Communications Networks". IEEE Transactions of the Professional Technical Group on Communications Systems.

Bashir, I. (2017). "Mastering Blockchain". Packt Publishing.

BitFury Group, J. G. (2015a). "Public versus Private Blockchains - Part 1: Permissioned Blockchains". Disponível em: https://bitfury.com/content/downloads/public-vsprivate-pt1-1.pdf. Acesso em maio, 2019.

BitFury Group, J. G. (2015b). "Public versus Private Blockchains - Part 2: Part 2: Permissionless Blockchains". Disponível em: https://bitfury.com/content/downloads/public-vs-private-pt2-1.pdf. Acesso em maio, 2019.

Blockcerts (2016). "What we learned from designing an academic certificates system on the blockchain". Disponível em: https://medium.com/mit-media-lab/what-welearned-from-designing-anacademic-certificates-system-on-the-blockchain34ba5874f196. Acesso em maio, 2019.

Burnett, A. (2018). "2 technologies you can't ignore: JavaScript and blockchain". Disponível em: https://www.pluralsight.com/resource-center/guides/javascript-andblockchain. Acesso em abril, 2019.

Buterin, V. (2013). "Ethereum: A Next-Generation Smart Contract and Decentralized Application Platform". Disponível em: https://github.com/ethereum/wiki/wiki/White-Paper. Acesso em abril, 2019. 
Chopra, K.; Gupta, K.; Lambora, A. (2019) "Proof of Existence Using Blockchain". International Conference on Machine Learning, Big Data, Cloud and Parallel Computing (COMITCon), Faridabad, India, pp. 429-431, DOI: 10.1109/COMITCon.2019.8862441.

Costa, R.; Faustino, D.; Lemos, G.; Queiroga, A.; Djohnnatha, C.; Alves, F.; Lira, J.; Pires, M. (2018). "Uso Não Financeiro de Blockchain: Um Estudo de Caso Sobre o Registro, Autenticação e Preservação de Documentos Digitais Acadêmicos". Disponível em: https://sol.sbc.org.br/index.php/wblockchain/article/view/2356. Acesso em maio, 2019.

Crockford, D. (2008). “JavaScript: The Good Parts”. O’Reilly Media, Inc.

Ellervee, A.; Matulevicius, R.; Mayer, N. (2017). "A Comprehensive Reference Model for Blockchain-based Distributed Ledger Technology". Disponível em: http://ceurws.org/Vol-1979/paper-09.pdf. Acesso em abril, 2019.

Gamma, E.; Helm, R.; Johnson, R.; Vlissides, J. (1995). "Design Patterns: Elements of Reusable Object-oriented Software". Addison-Wesley Longman Publishing Co., Inc., Boston, MA, USA.

Huotari, K.; Hamari, J. (2017). "A definition for gamification: anchoring gamification in the service marketing literature". Electronic Markets, 27(1):21-31. Disponível em: https://link.springer.com/article/10.1007/s12525-015-0212-z. Acesso em abril, 2019.

Kano, Y.; Nakajima, T. (2017). “An alternative approach to blockchain mining work for making blockchain technologies fit to ubiquitous and mobile computing environments". In 2017 Tenth International Conference on Mobile Computing and Ubiquitous Network (ICMU), pages 1-4. Disponível em: https://ieeexplore.ieee.org/document/8330097. Acesso em maio, 2019.

Moise, D.; Cruceru, A. F. (2014). "The Use of Gamification in Events Marketing". International Journal of Economic Practices and Theories. Disponível em: https://www.academia.edu/24702554/The_Use_of_Gamification_in_Events_Marke ting. Acesso em maio, 2019.

Nakamoto, S. (2008). “Bitcoin: A Peer-to-Peer Eletronic Cash System”. Disponível em: https://bitcoin.org/en/bitcoin-paper. Acesso em maio, 2019.

Narayanan, A; Bonneau, J.; Felten, E. W.; Miller, A.; Goldfeder, S. (2016). "Bitcoin and Cryptocurrency Technologies - A Comprehensive Introduction". Princeton University Press.

OriginalMy.COM (2019). “Original My Blockchain”. Disponível em: https://originalmy.com. Acesso em maio, 2019.

Pereira, C. R. (2018). "Node.js - Aplicações Web real-time com Node.js". Casa do Código.

Proof Of Existence (2019). Proof of Existence. Disponível em: https://docs.proofofexistence.com/\#/. Acesso em maio, 2019.

Tschorsch, F.; Scheuermann, B. (2016). "Bitcoin and Beyond: A Technical Survey on Decentralized Digital Currencies". IEEE Communications Surveys Tutorials, 
112: Cadernos do IME : Série Informática : Vol. 45 Dezembro 2020

18(3):2084-2123. Disponível em: https://ieeexplore.ieee.org/document/7423672. Acesso em maio, 2019.

Ulrich, F. (2017). “Bitcoin: a moeda na era digital”. LVM Editora. Walport. 